\title{
Multi-Drug Resistance Pattern of Lactose Non-Fermenting Escherichia coli as Causative Agent of Urine Tract Infections in Luanda, Angola
}

\author{
Aleksey Shatalov \\ Laboratory of Microbiology at Luanda Medical Center, Luanda, Angola \\ Email: alshatalov@gmail.com
}

How to cite this paper: Shatalov, A. (2019) Multi-Drug Resistance Pattern of Lactose Non-Fermenting Escherichia coli as Causative Agent of Urine Tract Infections in Luanda, Angola. Open Journal of Medical Microbiology, 9, 1-7.

https://doi.org/10.4236/ojmm.2019.91001

Received: December 21, 2018

Accepted: January 25, 2019

Published: January 28, 2019

\section{Copyright $\odot 2019$ by author(s) and} Scientific Research Publishing Inc. This work is licensed under the Creative Commons Attribution International License (CC BY 4.0).

http://creativecommons.org/licenses/by/4.0/

\begin{abstract}
This prospective study was carried out to assess the sensitivity and resistance pattern of lactose non-fermenting Escherichia coli from July 2018 to December 2018 in the Laboratory of Microbiology at Luanda Medical Center, Angola. Out of 1170 patient, a total of 120 urine specimens infected with Escherichia coli $\left(>10^{5} \mathrm{CFU} / \mathrm{ml}\right)$ were collected according to the routine protocol of urinalysis. Among these 120 isolates, 25 (21\%) isolates were determined as "atypical", lactose non-fermenting E. colis trains. The twenty-five lactose non-fermenting Escherichia coli strains isolated from urine samples in Luanda Medical Center were declared as Multiple Drugs-Resistant strains with high resistance to Cefalexine (100\%), Cefuroxime (100\%), Ceftriaxone (92\%), Gentamycin (92\%), Ciprofloxacin (72\%) and Amoxiciclin/Clavulanic (80\%). The alarming resistance level to the first-choice drugs for the treatment of urinary tract infections caused by non-fermentative lactose $E$. coli was observed.
\end{abstract}

\section{Keywords}

Escherichia coli, Multi-Drugs Resistance (MDR), Lactose Non-Fermenting, Urine Tract Infections, Colony Forming Unit (CFU)

\section{Introduction}

The Urinary tract infections (UTIs) are serious health affecting problems worldwide [1] [2]. The level of bacterial resistance to antibiotics is growing from year to year and is becoming one of the main problems in the world [3]. This is especially true for the developing countries including African continent where 
self-medication, overuse and misuse of antibiotics leads to the emergence of multi drug resistant (MDR)bacteria [4] [5]. E. coli is the most common and predominated bacteria causing UTIs in human beings. E. coli is facultative anaerobic, Gram-negative bacilli ferments lactose to produce hydrogen sulphide. Historically been reported that up to $10 \%$ of $E$. coli can be "atypical" non-lactose fermenting. Lactose non-fermenting E. coli has a number of virulence factors and exhibit antibiotic resistance [6]. Now there is not a lot of data about lactose non-fermenting $E$. coli as the etiological agent of urinary tract infection, while the Gram-negative, non-fermenting multi-resistant bacilli make the treatment of these organisms very difficult and expensive [7]. According to the Antimicrobial Resistance Global Report of WHO, the data about antibiotic resistance obtained from the African countries is still not enough [1]. The aim of this study was conducted to determine the antibiotic resistance pattern of lactose non-fermenting Escherichia coli.

\section{Subjects and Methods}

\subsection{Setting}

This prospective study was carried out to assess the sensitivity and resistance pattern of lactose non-fermenting Escherichia coli from July 2018 to December 2018 in the Laboratory of Microbiology at Luanda Medical Center, Angola.

\subsection{Sampling}

Bacteria were isolated according to the routine protocol of urinalysis using MacConkey/CLED HY-Uritest and chromogenic media (Hy-Labs, Israel). The Uritest paddle was dipped into the aseptically collected urine sample and removed immediately. The paddle was transferred into the tube and cap was screwed back loosely, to allow for free transfer of atmosphere. The tube was incubated $18-24$ hours at $37^{\circ} \mathrm{C}$. Test yielding $\geq 10^{5} \mathrm{CFU} / \mathrm{ml}$ are regarded as Positive. Additionally, the $0.01 \mathrm{~mL}$ of urine sample was inoculated on CHROME Orientation agar (HyLabs Ltd) by spread plate technique. The determination of lactose non-fermenter strains was using by observation colonies on MacConkey agar and Cystine Lactose Electrolyte Deficient (CLED) Agar. The lactose non-fermenting E. coli colonies were colorless on McConkey agar and colorless to blue on CLED agar. The isolated bacteria were then identified by using Gram Stain and their biochemical characteristics using Remelrap ID system kits.

\subsection{Antimicrobial Susceptibility Testing}

Antibiotic susceptibility was determined using the disc diffusion method on Mueller Hinton agar according to the Guidelines of the Clinical Laboratory Standards Institute (GCLSI). Different families of antibiotics (discs obtained from OXOID) were used in this study included Cephems; Beta-lactam + inhibitor, Cephalosporines (1st, 2nd and 3rd generations); Fluoquinolones; Tetracyclins; Folate pathway inhibitors; Nitrofurans; Aminoglycosides; Monobactams 
and Carbapenemes. The diameter of the inhibition zone formed around the disc was measured and compared to the critical values of each antibiotic disc (according to CLSI) to qualify the target bacteria as sensitive or resistant. MDR bacteria are defined as resistant to at least three different classes of antibiotics. Multi Resistant strains were according to the Center for Disease prevention and Control [8] [9] [10].

\section{Results}

\subsection{Samples}

Between July 2018 and December 2018, 187 positive urine samples from 1170 out-patients were collected at Luanda Medical Center. Out of them, 120 (64\%) urine specimens were infected with E. coli $\left(>10^{5} \mathrm{CFU} / \mathrm{ml}\right) .107$ (89\%) positive sample were belong to females and $13(11 \%)$ were belong to males. Among these 120 isolates, 25 (21\%) isolates were determined as atypical, lactose non-fermenting E. coli. The twenty two (88\%) lactose non-fermenting E. coli we isolated from female urine samples and 3 (12\%) strains were isolated from male samples. The average patient with UTI caused by lactose non-fermenting E. coli were $41 \pm 14$ years for males and $40 \pm 14$ for females.

\subsection{Antibiotic Resistance}

The comparison of antibiotic resistance rates was performed specifically for lactose non-fermenting E. coli (LNFEC) and lactose fermenting $E$. coli (LFEC) in (Table $1 \&$ Table 2). All of lactose non-fermenting E. coli isolates were Multiple Drug Resistant and exhibited the highest resistance to Cefalexine (100\%), Cefuroxime (100\%), Ceftriaxone (92\%), Trimethoprim/Sulfamethoxazole (92\%), Doxycycline (92\%), Gentamicin (92\%) followed by high level of resistance to Amoxicillin/Clavulanic acid (80\%), Ciprofloxacin (72\%) and low level of resistance to Nitrofurantoin (16\%). It was not found lactose non-fermenting E. coli isolates resistant to Imipinem, Piperacilin/Tazobactam and Amikacin (Table 1).

While the lactose fermenting $E$. coli isolates exhibited the intermediate level of resistance to Trimethoprim/Sulfamethoxazole (55\%), Doxycycline (78.4\%) followed by low level of resistance to Ciprofloxacin (21\%), Amoxicillin/Clavulanic acid (18\%), Cefalexine (14\%) Gentamicin (8\%), Nitrofuratoin (5\%), Cefuroxime (3\%), Ceftriaxone (1\%). It was not found lactose fermenting E. coli isolates resistant to Piperacilin/Tazobactam, Amikacin, Aztreonam and Imipinem (Table 2).

\subsection{Multi-Drug Resistance}

$100 \%$ of the lactose non-fermenting $E$. coli isolates were found to be multidrug resistant while $21 \%$ lactose fermenting $E$. coli isolates were MDR.

\section{Discussion}

Urine tract infection is one of the most common infections in the world [1] [2]. 
Table 1. Antibiotic resistance rates for Lactose Non-fermenting E. coli (LNFEC) isolates.

\begin{tabular}{cccccccc}
\hline Antibiotic subclass & Antibiotic & \multicolumn{2}{c}{ Resistant } & Intermediate & \multicolumn{2}{c}{ Sensitive } \\
\hline & & $\mathrm{n}$ & $\%$ & $\mathrm{n}$ & $\%$ & $\mathrm{n}$ & $\%$ \\
\hline \multirow{2}{*}{ Beta-lactam + inhibitor } & Amoxicillin/clavulanic acid & 20 & 80 & 0 & 0 & 5 & 20 \\
& Piperacilin/tazobactam & 0 & 0 & 0 & 0 & 25 & 100 \\
Cephalosporin I & Cefalexine & 25 & 100 & 0 & 0 & 0 & 0 \\
Cephalosporin II & Cefuroxime & 25 & 100 & 0 & 0 & 0 & 0 \\
Cephalosporin III & Ceftriaxone & 22 & 92 & 0 & 0 & 27 & 8 \\
Fluoroquinolones & Ciprofloxacin & 18 & 72 & 0 & 0 & 7 & 28 \\
Tetracyclines & Doxycyline & 22 & 92 & 0 & 0 & 3 & 8 \\
Folate pathway inhibitors & Trimethoprim/sulfamethoxazole & 22 & 92 & 0 & 0 & 3 & 8 \\
Aminoglycosides & Gentamicin & 22 & 92 & 0 & 0 & 3 & 8 \\
Nitrofurans & Amikacin & 0 & 0 & 0 & 0 & 25 & 100 \\
Monobactam & Nitrofurantoin & 5 & 20 & 0 & 0 & 20 & 80 \\
Carbapenemes & Aztreonam & 12 & 48 & 5 & 20 & 8 & 32 \\
\hline & Imipinem & 0 & 0 & 0 & 0 & 25 & 100 \\
\hline
\end{tabular}

Table 2. Antibiotic resistance rates for Lactose fermenting E. coli (LFEC) isolates.

\begin{tabular}{|c|c|c|c|c|c|c|c|}
\hline \multirow[t]{2}{*}{ Antibiotic subclass } & \multirow[t]{2}{*}{ Antibiotic } & \multicolumn{2}{|c|}{ Resistant } & \multicolumn{2}{|c|}{ Intermediate } & \multicolumn{2}{|c|}{ Sensitive } \\
\hline & & $\mathrm{n}$ & $\%$ & $\mathrm{n}$ & $\%$ & $\mathrm{n}$ & $\%$ \\
\hline \multirow{2}{*}{ Beta-lactam + inhibitor } & Amoxicillin/clavulanic acid & 18 & 18 & 9 & 9 & 73 & 73 \\
\hline & Piperacilin/tazobactam & 0 & 0 & 0 & 0 & 95 & 100 \\
\hline Cephalosporin I & Cefalexine & 14 & 15 & 65 & 68 & 15 & 17 \\
\hline Cephalosporin II & Cefuroxime & 3 & 3 & 17 & 18 & 75 & 79 \\
\hline Cephalosporin III & Ceftriaxone & 1 & 1 & 0 & 94 & 0 & 99 \\
\hline Fluoroquinolones & Ciprofloxacin & 20 & 21 & 3 & 3 & 72 & 76 \\
\hline Tetracyclines & Doxycyline & 43 & 45 & 1 & 1 & 51 & 54 \\
\hline Folate pathway inhibitors & Trimethoprim/sulfamethoxazole & 52 & 55 & 1 & 1 & 42 & 44 \\
\hline \multirow{2}{*}{ Aminoglycosides } & Gentamicin & 8 & 8 & 0 & 0 & 87 & 92 \\
\hline & Amikacin & 0 & 0 & 0 & 0 & 95 & 100 \\
\hline Nitrofurans & Nitrofurantoin & 4 & 5 & 1 & 1 & 90 & 95 \\
\hline Monobactam & Aztreonam & 0 & 0 & 0 & 0 & 95 & 100 \\
\hline Carbapenemes & Imipinem & 0 & 0 & 0 & 0 & 95 & 100 \\
\hline
\end{tabular}

E. coli is the most common and predominated causative bacteria in urine tract infections. The level of bacterial resistance to antibiotics is growing from year to year and is becoming one of the main problems in the world [3] [11]. Particularly concerned about the increase multidrug resistance level of lactose non-fermenting gram negative rods. The lactose non-fermenting bacilli possess several different 
mechanisms of resistance that makes treatment of these organisms difficult and expensive [6] [7]. This is especially true for the developing countries including African continent where improper medication, self-medication, overuse and misuse of antibiotics leads to the emergence of MDR bacteria [4].

Our study has showed that E. coli were implicated in $64 \%$ of all Urine Tract Infection pathogens. This rate is similar to results reported from Maroco (63\%) [12], Ethiopia (60\%) [13], but higher from studies in Equatorial Guinea (55\%) [14], Nigeria (37\%) [15] and Ghana (37\%) [16]. The rate of lactose non fermenting E. coli urinary isolates from this study was $21 \%$, which is similar to the study authored by Chang (about 20\%) [17] and more higher than studies of Bhat (about 13\%) [18], Bajapai (4\%) [19].

Our study revealed $72 \%$ lactose non-fermenting E. coli isolates resistant to Ciprofloxacin. This result agreed with study of Chang [17] who recorded $66.7 \%$ resistance to Ciprofloxacin and was higher than results published by Yaratha (30\% resistance to Ciprofloxacin) [20]. Our study has showed that "atypical" lactose non-fermenting $E$. coli isolated were highly resistant to a broad spectrum of antibiotics: Cefalexine (100\%), Cefuroxime (100\%), Ceftriaxone (92\%), Trimethoprim/Sulfamethoxazole (92\%), Doxycycline (92\%), Gentamicin (92\%), Amoxicillin/Clavulanic acid (80\%), Ciprofloxacin (72\%). These results demonstrate the highest level resistance of lactose non-fermenting bacteria in comparison with the resistant pattern of "common" lactose fermenting $E$. coli isolates as observed in this study (Figure 1). Our date is partially agrees with some studies in Nigeria [21], Maroco [12] and Kenya [22] were the similar results were received.

\section{Conclusion}

Our study has shown the high percentage (21\%) presence of the "atypical" lactose

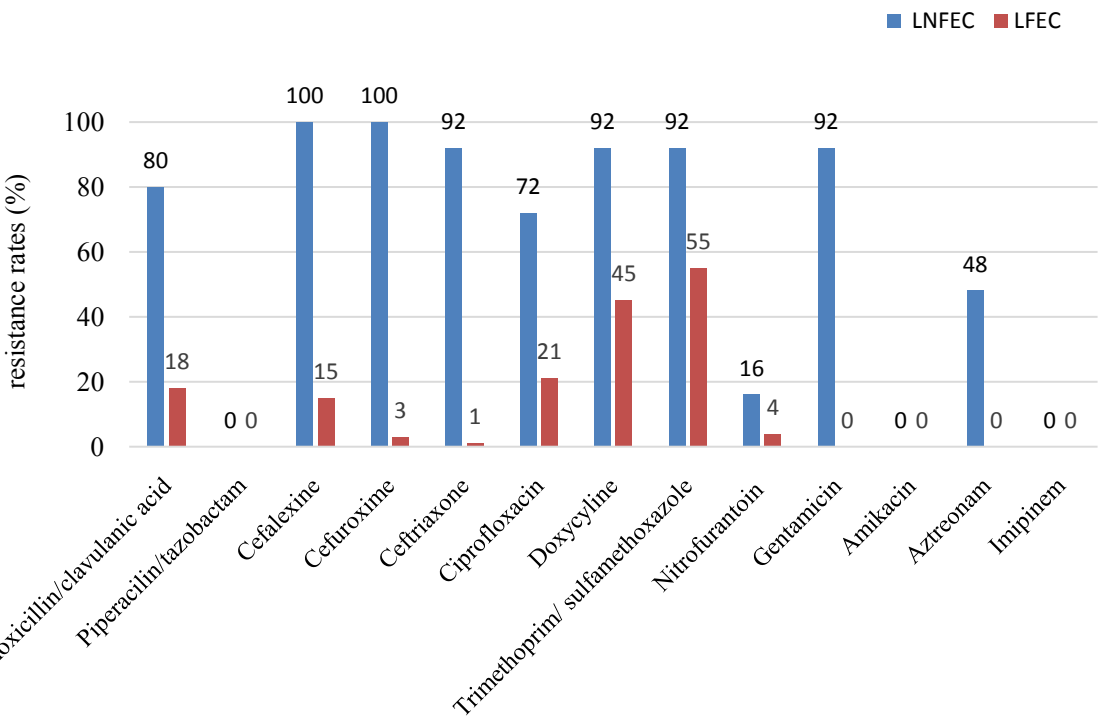

Figure 1. Antibiotic resistance rates (\%) for Lactose Non-Fermenting E. coli (LNFEC) and Lactose Fermenting E. coli (LFEC) isolates. 
non-fermenting $E$. coli causing urinary tract infections. The alarming level of multi-drug resistance to the first-choice drugs for the treatment of UTIs caused by lactose non-fermenting E. coli was detected. To our knowledge, this is the first study determined the antibiotic resistance pattern of in UTIs in Angola.

\section{Acknowledgements}

The author expresses gratitude to Dr. Michael Averbukh, Dr. Ziv Maianski, Dr. Aaron Cohen and all staff of the Luanda Medical Center for their help and support.

\section{Conflicts of Interest}

The author declares that he has no competing interests.

\section{References}

[1] WHO (2014) Antimicrobial Resistance: Global Report on Surveillance. http://www.who.int/drugresistance/documents/surveillancereport/en/

[2] Foxman, B. (2010) The Epidemiology of Urinary Tract Infection. Nature Reviews Urology, 7, 653-660. https://doi.org/10.1038/nrurol.2010.190

[3] Schito, G., Naber, K., Botto, H., et al. (2009) The ARESC Study: An International Survey on the Antimicrobial Resistance of Pathogens Involved in Uncomplicated Urinary Tract Infections. International Journal of Antimicrobial Agents, 34, 407-413. https://doi.org/10.1016/j.ijantimicag.2009.04.012

[4] Tadesse, B.T., Ashley, E.A., Ongarello, S., et al. (2017) Antimicrobial Resistance in Africa: A Systematic Review. BMC Infectious Diseases, 17, 616. https://doi.org/10.1186/s12879-017-2713-1

[5] Essack, S.Y., Desta, A.T., Abotsi, R.E., et al. (2017) Antimicrobial Resistance in the WHO African Region: Current Status and Roadmap for Action. Journal of Public Health, 39, 8-13.

[6] Kaczmarek, A., Skowron, K., Budzyńska, A., et al. (2017) Virulence Genes and Antimicrobial Susceptibility of Lactose-Negative and Lactose-Positive Strains of Escherichia coli Isolated from Pregnant Women and Neonates. Folia Microbiologica, 62, 363-371. https://doi.org/10.1007/s12223-017-0506-y

[7] McGowan, J.E. (2006) Resistance in Nonfermenting Gram-Negative Bacteria: Multidrug Resistance to the Maximum. American Journal of Infection Control, 34, 29-37. https://doi.org/10.1016/j.ajic.2006.05.226

[8] http://www.cdc.gov/hicpac/pdf/guidelines/MDROGuideline2006.pdf

[9] Magiorakos, A., Srinivasan, A., Carey, R., et al. (2012) Multidrug-Resistant, Extensively Drug-Resistant and Pandrug-Resistant Bacteria: An International Expert Proposal for Interim Standard Definitions for Acquired Resistance. Clinical Microbiology and Infection, 18, 268-281. https://doi.org/10.1111/j.1469-0691.2011.03570.x

[10] Clinical Laboratory Standards Institute (2015) Performance Standards for Antimicrobial Susceptibility Testing. M100-S23:35(3).

[11] Cohen, M.L. (2000) Changing Patterns of Infectious Disease. Nature, 406, 762-767. https://doi.org/10.1038/35021206

[12] Bourjilat, F., Bouchrif, B., Dersi, N., et al. (2011) Emergence of Extended-Spectrum 
Beta-Lactamases-Producing Escherichia coli in Community-Acquired Urinary Infections in Casablanca, Morocco. The Journal of Infection in Developing Countries, 5, 850-855.

[13] Abejew, A., Denboba, A. and Mekonnen, A. (2014) Prevalence and Antibiotic Resistance Pattern of Urinary Tract Bacterial Infections in Dessie Area, North-East Ethiopia. BMC Reseach Notes, 7, 687. https://doi.org/10.1186/1756-0500-7-687

[14] Shatalov, A. (2015) Prevalence and Antibiotic Resistance Pattern of Escherichia coli and Klebsiella pneumoniae in Urine Tract Infections at the La Paz Medical Center, Malabo, Equatorial Guinea. Open Journal of Medical Microbiology, 5, 177-183. https://doi.org/10.4236/ojmm.2015.54022

[15] Iregbu, K. and Princewill, P. (2013) Urinary Tract Infection in a Tertiary Hospital in Abuja, Nigeria. African Journal of Clinical and Experimental Microbiology, 14, 167-173. https://doi.org/10.4314/ajcem.v14i3.9

[16] Obirikorang, C., Quaye, L., Bio, F., et al. (2012) Asyptomatic Bacteriuria among Pregnan Women Attending Antenatal Clinic at the University Hospital, Kumasi, Ghana. Journal of Medicine and Biomedical Sciences, 1, 38-44.

[17] Chang, J., Yu, J., Lee, H., et al. (2014) Prevalence and Characteristics of Lactose Non-Fermenting Escherichia coli in Urinary Isolates. Journal of Infection and Chemotherapy, 20, 738-740. https://doi.org/10.1016/j.jiac.2014.07.005

[18] Bhat, K.G. and Bhat, M.G. (1995) Atypical Escherichia coli in Urinary Tract Infection. Tropical Doctor, 5, 127. https://doi.org/10.1177/004947559502500313

[19] Bajpai, T., Pandey, M., Varma, M., et al. (2016) Importance of Identification of Lactose Nonfermenting Escherichia coli and Their Prevalence in Urinary Isolates. CHRISMED Journal of Health and Research, 3, 288-290. https://doi.org/10.4103/2348-3334.190581

[20] Yaratha, G., Perloff, S. and Changala, K. (2017) Lactose vs. Non-Lactose Fermenting E. coli: Epidemiology, Clinical Outcomes, and Resistance. Open Forum Infectious Diseases, 4, 589-590. https://doi.org/10.1093/ofid/ofx163.1546

[21] Oladeinde, B.H., Omoregie, R., Olley, M., et al. (2011) Urinary Tract Infection in a Rural Community of Nigeria. North American Journal of Medical Sciences, 3, 75-77. https://doi.org/10.4297/najms.2011.375

[22] Kariuki, S., Revathi, G., Corkill, J., et al. (2007) Escherichia coli from Community-Acquired Urinary Tract Infections Resistant to Fluoroquinolones and Extended-Spectrum Beta-Lactams. The Journal of Infection in Developing Countries, 1, 257-262. https://doi.org/10.3855/jidc.361 